\title{
A REVIEW OF GRB COUNTERPART SEARCHES
}

\author{
C. KOUVELIOTOU \\ Universities Space Research Association \\ ES-84, NASA/MSFC, Huntsville, Alabama 35812, USA
}

\section{Introduction}

Gamma-Ray Burst (GRB) research has been recently revitalized with exciting new results that have effectively started a new era in this thirty year old field, marked by the launch of the Italian-Dutch satellite BeppoSAX [1] on April 1996.

In the following, I will briefly (due to the limited space available) describe the searches and their results for each of the seven GRBs that have been rapidly followed-up in multiwavelength observations. As BeppoSAX, CGRO and RXTE are fully operational, we hope that the GRB counterpart sample will increase significantly within the next year, thus providing new insights to the properties of their parent population.

\section{GRB Counterpart Searches}

GRB970111: The event was detected with three satellites (BeppoSAX, BATSE, Ulysses). The original WFC error box (10' in diameter) [5] was observed with the NFIs $\sim 21$ hours after the burst trigger, and three Xray sources were detected $[3,32]$. A variable radio source was found within this error box [9] that coincided with one of the X-ray sources. A refined WFC error box [20] reduced the source location to a $3^{\prime}$ radius region, which although well within the original location, clearly excluded all X-ray and the radio source as the GRB counterparts. The radio non-detection may be used to set limits to GRB distances [12].

GRB970228: This is the first counterpart detection of a GRB after 30 years of searches [33]. The burst was detected and located with BeppoSAX [6], and it was observed with optical telescopes $\sim 21$ hours after detection. Eight hours after the burst trigger, the NFIs detected an X-ray source associated with the WFC position, which decayed with an -1.1 power law index 
until the next NFI observation on 3 March 1997 [7]. Optical observations of the WFC error region made on 28 February and 8 March 1997 revealed a counterpart $[16,33]$ that was within the $0.75 \operatorname{arcmin}^{2}$ area defined by the WFC, NFI and the IPN annuli between Ulysses/SAX and Ulysses/WIND [19]. Subsequent optical observations on 13 March 1997 with ground-based telescopes revealed the existence of a 'fuzz' at the position of the transient [17]; the 'fuzz' was resolved with the Hubble Space Telescope (HST) Planetary Camera observations (on 26 March and 7 April 1997) into a point source and an extended source [29]. Claims of proper motion detection and intensity changes of the extended source [4] were not corroborated with a second HST observation on 4 September 1997, that detected the point source at the same position and the extended source at the same intensity [11].

GRB970402: This event was detected only with BeppoSAX; WFC located it to $3^{\prime}$ accuracy and the NFIs were repointed. A very weak X-ray source was detected that was not observed during a second pointing [28]. No optical/radio counterpart was found for this source.

GRB970508: H. Bond [2] discovered the optical counterpart associated with GRB970508 using the 36" telescope at Kitt Peak. It was subsequently observed with the Palomar [8] and with the Keck [23] telescopes; the latter. observation led to the first limits for a GRB redshift, ie., $0.835<z<2.3$. The lower limit is associated with the detection of absorption lines in the optical spectrum of the counterpart; the upper limit is set from the absence of the Lyman- $\alpha$ absorption features in the spectrum [23]. HST observations of the source did not detect any nebulosity asssociated with the burst counterpart [27]. GRB970508 is the only event so far with an identification of a radio counterpart [10]. The VLA data show rapid fluctuations during the first two weeks, that decline thereafter. The simplest explanation of the fluctuations is that they are due to scintillation caused by the interstellar medium in our Galaxy [10]. The decline of their amplitude is a consequence of the increase of the angular size of the source; initial estimates of the source size are $\sim 3 \mu$ arcsec. Such a source size is consistent with the fireball expansion models that are prevalent in the field these days [22].

GRB970616: This is the first afterglow detected with the PCA scanning of a BATSE error box [21]. The RXTE/PCA source intensity was $\sim 0.5$ $\mathrm{mCrab}$ and was located with $1^{\prime}$ accuracy [21]; subsequent observations of the RXTE error box with ASCA revealed four X-ray sources within the $P C A$ region [24]. ROSAT observations have shown that two of these are variable [13], which makes a choice for an X-ray counterpart uncertain. No optical/radio counterpart was detected for this event.

GRB970815: The burst was detected with the RXTE/All Sky Monitor(ASM) [30]; its error box was subsequently scanned with the PCA, but 
no X-ray afterglow was discovered. The ASM error box was observed with ASCA and ROSAT but no counterparts were found [25, 14].

GRB970828: This is the second burst that was detected with the ASM [31]. The source region was observed with ASCA and ROSAT, which detected a declining X-ray afterglow within its error box $[26,15]$. No opti$\mathrm{cal} /$ radio counterpart was found to coincide with the ASCA/ROSAT source [18].

TABLE 1. Summary of search results of GRB counterparts

\begin{tabular}{|c|c|c|c|c|c|c|}
\hline GRB & $\begin{array}{l}\text { Prompt } \\
\text { X-rays } \\
\text { (Crab) }\end{array}$ & $\begin{array}{l}\gamma \text {-rays } \\
\mathrm{erg} / \mathrm{cm}^{2}\end{array}$ & $\begin{array}{l}\text { Secondary } \\
\text { X-rays } \\
\mathrm{erg} / \mathrm{cm}^{2} \mathrm{~s}\end{array}$ & Optical & $\begin{array}{l}\text { Radio } \\
\text { mJy }\end{array}$ & Comments \\
\hline 970111 & $4.00^{a}$ & $6 \times 10^{-5, b}$ & - & - & - & Very intense GRB \\
\hline 970228 & $2.30^{a}$ & $10^{-6, c}$ & $3 \times 10^{-12, d}$ & $\begin{array}{l}\mathrm{V}=21.3 \\
\mathrm{I}=20.6\end{array}$ & - & $\begin{array}{l}\text { First optical } \\
\text { counterpart with } \\
\text { nebulosity }\end{array}$ \\
\hline 970402 & $0.46^{a}$ & $?$ & $2 \times 10^{-13, d}$ & $\begin{array}{l}V>22.5 \\
R>21.0\end{array}$ & - & $\begin{array}{l}\text { Very weak NFI } \\
\text { source }\end{array}$ \\
\hline 970508 & $1.00^{a}$ & $3 \times 10^{-6, b}$ & $6 \times 10^{-13, d}$ & $\begin{array}{l}V=20.5 \\
R=19.8\end{array}$ & $0.43^{e}$ & $\begin{array}{l}\text { First redschift } \\
\text { Radio source size: } \\
<10^{17} \mathrm{~cm}\end{array}$ \\
\hline 970616 & $?$ & $4 \times 10^{-5, b}$ & $1 \times 10^{-11, f}$ & $V>25.5$ & - & $\begin{array}{l}\text { Uncertain X-ray } \\
\text { counterpart }\end{array}$ \\
\hline 970815 & $2.00^{g}$ & $10^{-5, b}$ & $<10^{-13, h}$ & $\begin{array}{l}V>21.5 \\
R>23.0\end{array}$ & - & \\
\hline 970828 & $\sim 0.8^{g}$ & $7 \times 10^{-5, b}$ & $1 \times 10^{-11, f}$ & $R>25.0$ & - & Very intense GRB \\
\hline
\end{tabular}

${ }^{a}$ Detected with the Wide Field Cameras (WFC) on BeppoSAX ${ }^{b}$ Detected with the Burst And Transient Source Experiment (BATSE) on the Compton Gamma-Ray Observatory (CGRO) ${ }^{c}$ Detected with TGRS on WIND ${ }^{d}$ Detected with the Narrow Field Instruments (NFI) on BeppoSAX ${ }^{e}$ Detected with VLA ${ }^{f}$ Detected with the Proportional Counter Array (PCA) on the Rossi X-Ray Timing Explorer (RXTE) ${ }^{g}$ Detected with the All Sky Monitor (ASM) on RXTE ${ }^{h}$ Detected with ASCA

\section{SUMMARY}

Table 1 summarizes the GRB counterpart search results so far. The questions that are mostly asked today are: why some events do not have counterparts, what is the relation between detections in different wavelengths, what characteristics in GRB are correlated with the optical/X-ray afterglows. Although we have no definitive answers to any of these questions, 
simple explanations, such as large absorption from circumstellar dust in high to moderate redshifts could explain the lack of optical afterglows in several cases. Many more detections are needed, however, before we can establish a connection between GRB sources and their parent population.

\section{References}

1. Boella, G. et al. (1997) BeppoSAX, the wide band mission for X-ray astronomy, Astron. Astrophys. Suppl. Ser., 122, 299-399

2. Bond, H.E. (1997) IAU Circular No. 6654

3. Butler, R.G. et al. (1997) IAU Circular No. 6539

4. Caraveo, P. (1997) Talk presented at the 4th Compton Symposium in Williamsburg, VA, 27-30 April, 1997

5. Costa, E. et al., (1997a) IAU Circular No. 6533

6. Costa, E. et al., (1997b) IAU Circular No. 6572

7. Costa, E. et al. (1997c) Discovery of an X-ray afterglow associated with the $\gamma$-ray burst of 28 February 1997, Nature, 387, 783-785

8. Djorgovski, S.G. et al. (1997) The optical counterpart to the $\gamma$-ray burst GRB970508, Nature, 387, 876-878

9. Frail, D.A. et al. (1997a) Radio monitoring of the 1997 January 11 Gamma-Ray Burst, Astrophys. J., 483, L91-L94

10. Frail, D.A., Kulkarni, S.R., Nicastro, L., Feroci, M., \& Taylor, G.B. (1997b) The radio afterglow from the $\gamma$-ray burst of 8 May 1997, Nature, 389, 261-263

11. Fruchter, A. et al., (1997) IAU Circular No. 6747

12. Galama, T.J. et al. (1997) Radio and optical follow-up observations and improved interplanetary network position of GRB970111, Astrophys. J., 486, L5-L9

13. Greiner, J. et al., (1997a) IAU Circular No. 6722

14. Greiner, J. (1997b) IAU Circular No. 6742

15. Greiner, J. et al., (1997c) IAU Circular No. 6757

16. Groot, P. et al., (1997a) IAU Circular No. 6584

17. Groot, P. et al., (1997b) IAU Circular No. 6588

18. Groot, P. et al., (1997c) Astrophys. J. Letters, in press

19. Hurley, K. et al., (1997) IAU Circular No. 6594

20. in't Zand, J. et al. (1997) IAU Circular: No. 6569

21. Marshall, F.E. et al. (1997) IAU Circular No. 6683

22. Meszaros, P. \& Rees, M. (1997) Optical and long-wavelength afterglow from gammaray bursts, Astrophys. J., 476, 232-237

23. Metzger, M.R. et al. (1997) Spectral constraints on the redshift of the optical counterpart to the $\gamma$-ray burst of 8 May 1997, Nature, 387, 878-880

24. Murakami, T., Fujimoto, R., Ueda, Y., \& Shibata, R. (1997a) IAU Circular No. 6687

25. Murakami, T., Ueda, Y., Ishida, M. , \& Fujimoto, R. (1997b) IAU Circular No. 6722

26. Murakami, T. et al. (1997c) IAU Circular No. 6732

27. Pian, E. et al. (1997) Astrophys. J., submitted

28. Piro, L. et al., (1997) IAU Circular No. 6617

29. Sahu, K. et al. (1997) The optical counterpart to $\gamma$-ray burst GRB970228 observed using the Hubble Space Telescope, Nature, 387, 476-478

30. Smith, D.A, Levine, A., Morgan, E.H., \& Wood, A. (1997a) IAU Circular No. 6718

31. Smith, D.A, Levine, A., Remillard, R., \& Wood, A. (1997b) IAU Circular No. 6728

32. Voges, W., Boller, T., \& Greiner, J. (1997) IAU Circular No. 6539

33. Van Paradijs, J. et al. (1997) Transient optical emission from the error box of the gamma-ray burst of 28 February 1997, Nature, 387, 686-689 